\title{
Superhydrophobicity Due to the Hierarchical Scale Roughness of PDMS Surfaces
}

\author{
Barbara Cortese,* Stefania D’Amone, Michele Manca, Ilenia Viola, Roberto Cingolani, and \\ Giuseppe Gigli
} NNL, National Nanotechnology Laboratories of CNR-INFM, Distretto Tecnologico, Università del Salento,
Via Arnesano 16, 73100, Lecce, Italy

Received September 8, 2007. In Final Form: November 22, 2007

\begin{abstract}
Wettability control has been widely investigated in the last decades for technological applications such as microfluidic devices and self-cleaning surfaces by modifying both the chemical composition and the geometric structure of the surfaces. Inspired by the typical morphology of superhydrophobic leaves (such as lotus leaves), we have developed a dual-scale roughness, micro- and nanosized, on polydimethylsiloxane (PDMS) surfaces. By combining different geometric parameters and plasma treatment conditions, the structures were controlled hierarchically, at different independent length scales. Both the microsized replicated pillars and the nanosized etched posts tuned the wettability of the PDMS surfaces in a very simple way, up to contact angles of $170^{\circ}$. Furthermore, changes in the influence of micro- and nanoscale geometrical structures were investigated. Hysteresis and contact angles of water droplets are evaluated as a combined effect of micropillars and a superimposed roughness, resulting in high advancing contact angles and low sliding angles.
\end{abstract}

\section{Introduction}

The emerging interest in self-cleaning surfaces ${ }^{1,2}$ along with drop motion in microfluidic devices can be attributed to several practical applications ${ }^{3-7}$ and currently remains a technological challenge. Indeed, water repellency has potential industrial importance, ${ }^{8,9}$ such as contamination prevention, biocompatibility, anti-oxidation, ${ }^{10}$ minimization of the resistance to flow in microfluidic applications, ${ }^{11,12}$ and lab-on-a-chip systems. Nature has inspired many researchers ${ }^{13}$ in developing superhydrophobic surfaces by manipulating both topological and chemical properties. It is renowned that the lotus effect ${ }^{14}$ is characterized by the combination of micrometer-sized papillae and nanometer-sized branchlike protrusions and by chemical effects owing to a layer of epicuticular wax that covers the papillae. Therefore, the principles required to engineer a surface involve materials that exhibit a hierarchical roughness and low-energy surface properties. ${ }^{15}$ Many previous studies have reported successful generation of hydrophobic surfaces utilizing top-down and bottom-up fabrication methods like photolithography, ${ }^{16}$ soft lithography, ${ }^{17}$

* Corresponding author. E-mail: barbara.cortese@unile.it.

(1) Nakajima, A.; Hashimoto, K.; Watanabe, T.; Takai, K.; Yamauchi, G.; Fujishima, A. Langmuir 2000, 16, 7044.

(2) Fürstner, R.; Barthlott, W.; Neinhuis, C.; Walzel, P. Langmuir 2005, 21, 956.

(3) Feng, L.; Song, Y.; Zhai, J.; Liu, B.; Xu, J.; Jiang, L.; Zhu, D. Angew. Chem. Int. Ed. 2003, 42, 800.;

(4) Shiu, Y.; Kuo, C. W.; Chen, P.; Mou, C. Y. Chem. Mater. 2004, 16, 561.

(5) Song, X.; Zhai, J.; Wang, Y.; Jiang, L. J. Phys. Chem. B 2005, 109, 4048.

(6) Wu, X.; Zheng, L.; Wu, D. Langmuir 2005, 21, 2665.

(7) Erbil, H.; Demirel, A.; Avci, Y. and Mert, O. Science 2003, 299, 1377, 31.

(8) Nakajima, A.; Hashimoto, K.; Watanabe, T. Monastsh. Chem. 2001,132,

(9) Quéré, D. Physica A 2002, 313, 32.

(10) Nakajima, A.; Fujishima, A.; Hashimoto, K.; Watanabe, T. Adv. Mater. 1999, 11,1365 .

(11) Kim, J. and Kim, C. J. Proceedings of the IEEE 15th International Conference MEMS, Las Vegas, NV, 2002; p 479

(12) Extrand, C. W. Langmuir 2003, 19, 3793-3796.

(13) Otten, A.; Herminghaus, S; Langmuir 2004, 20, (6), 2405.

(14) Barthlott, W.; Neinhuis, C. Planta 1997, 202, 1.

(15) Quéré, D. Nat. Maters 1 2002, p. 14

(16) Yoshimititsu, Z.; Nakajima, A.; Watanabe, T.; Hashimoto, K. Langmuir 2002, 18, 5818-5822. the Langmuir-Blodgett technique, ${ }^{18}$ and vapor deposition, ${ }^{19}$ all with the purpose to mimic a structure that resembles the lotus leaf by achieving microscale structures and a few to obtain a microhierarchical morphology. Plasma etching processes with surface plasma fluorination have been used to obtain nanoscale surface roughness on different substrates. ${ }^{20,21}$ Although many monorough surfaces have been realized, superhydrophobicity properties have been limited so far; thus, a dual roughened surface, mimicking lotus leaves, could hypothetically show more hydrophobic features. Moreover, it is reported that the controlled geometry of roughness of superhydrophobic surfaces, with regard to randomly roughened ones, allows better control over experimental parameters that contribute to determine superhydrophobicity. ${ }^{22}$ Remarkable theoretical studies have been undertaken on structures with a double-scale roughness, though with very few experimental studies due to inherent difficulties in the realization of the specimens. A dual roughness has been reported by Zhu et al., ${ }^{23}$ although using two different materials, while Shirtcliffe et al. ${ }^{24}$ have modeled etched copper patterns surfaces with a combination of roughness and surface patterning. Kim et al. ${ }^{25}$ generated superhydrophobic surfaces by combining nanoscale surface roughening of $\mathrm{Si}$ with a hydrophobic a-C:H:Si:O coating. Jin et al. ${ }^{26}$ have created a superhydrophobic PDMS double structured surface by a one-step laser-cutting method. However,

(17) Chen, Y.; He, B.; Lee, J.; Patankar, N. A. J. Colloid Interface Sci. 2005 , $281,458-464$

(18) Gleiche, M.; Chi, L.; Gedig, E.; Fuchs, H. ChemPhysChem 2001, 3 , $187-191$.

(19) Gau, H.; Herminghaus, S.; Lenz, P.; Lipowsky, R. Science 1999, 283, $46-49$.

(20) Garra, J.; Long, T.; Currie, J.; Schneider, T.; White, R.; Paranjape, M. J. Vac. Sci. Technol. A, 2002, 20, 975.

(21) Tserepi, A.; Vlachopoulou, M.-E.; Gogolides, E. Nanotechnology 2006, 17, 3977-3983.

(22) Patankar, N. A. Langmuir 2003, 19, 1249-53.

(23) Zhu, L.; Xiu, Y.; Xu, J.; Tamirisa, P. A.; Hess, D. W.; Wong, C. P. Langmuir 2005, 21, 11208 .

(24) Shirtcliffe, N. J.; McHale, G.; Newton, M. I.; Perry, C. C. Langmuir 2005, 21, 937-943.

(25) Kim, T.-Y.; et al. Chem. Phys. Lett. 2007, 436, 199

(26) Jin, M.; Feng, X.; Xi, J.; Zhai, J.; Cho, K.; Feng, L.; Jiang, L. Macromol. Rapid Commun. 2005, 26, 1805. 


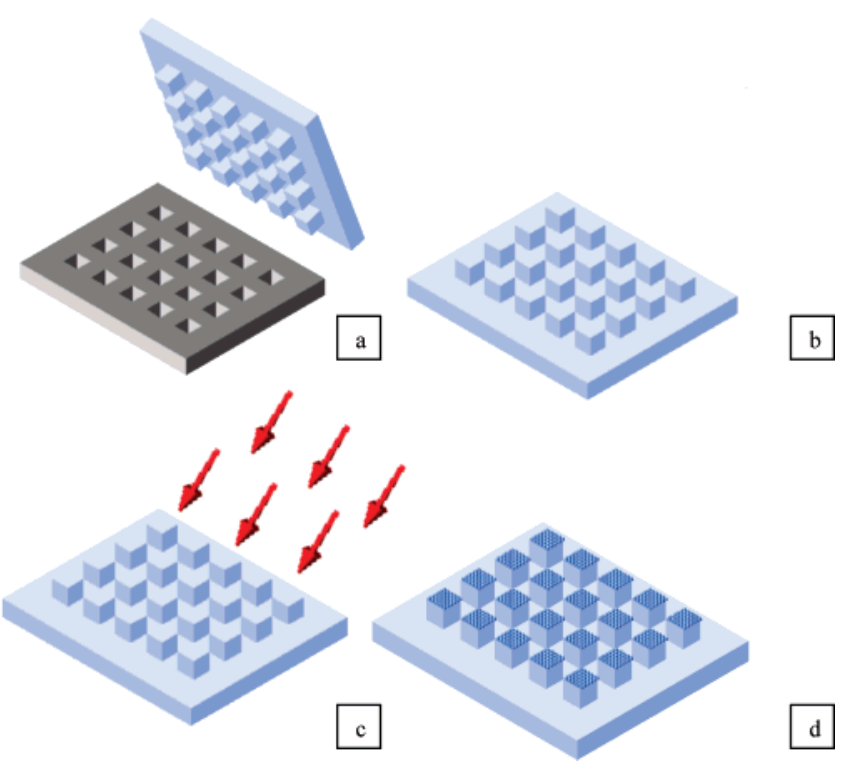

Figure 1. Schematic illustration of the fabrication process of the double-structured surfaces. Illustrations $\mathrm{a}$ and $\mathrm{b}$ represent the first step, where a PDMS replica of a prepatterned SU8 surface was obtained. Illustration c represents the second step due to $\mathrm{CF}_{4}$ plasma treatment of the replica. Illustration $\mathrm{d}$ represents the final dualstructured surface.

the methods mostly employed involved expensive materials or strict settings, with limitations in the applications.

In this study, we demonstrate an easy, low-cost, two-step procedure to fabricate superhydrophobic PDMS surfaces. We report that a monoscale roughness is not alone adequate to acquire hydrophobicity, showing that a superhydrophobic surface is best achieved with a hierarchical dual-scale roughness, as inspired by the lotus leaf. We fabricated various structures with mono and dual hierarchical roughness through microstructuring PDMS by simply replicating prepatterned structures and nanostructuring it by means of a $\mathrm{CF}_{4}$ plasma treatment. The two main steps of the procedure for the fabrication of dual-structured superhydrophobic surfaces are sketched in Figure 1.

The effects of the variation of geometrical parameters on superhydrophobicity, and the influence of introducing chemical and nanoscale roughness effects on microstructured PDMS pillars, have been investigated to show how a dual roughness structure affects superhydrophobic properties, exhibiting higher static contact angle (CA) and lower contact angle hysteresis (CAH) than monostructures. More importantly, contact angles up to $170^{\circ}$ and sliding angles of $1^{\circ}$ were reached.

\section{Theoretical Background}

According to theoretical analysis, the thermodynamic Young angle $(\theta)$ of a water droplet on a flat smooth surface is due to the balance between the relative interfacial contact areas in order to minimize the surface free energy, ${ }^{27}$ as shown in Figure 2. In general, the theoretical contact angle $\left(\theta_{\mathrm{w}}\right)$ on a rough surface, according to Wenzel's approach, ${ }^{28}$ is calculated considering the spaces left between the protrusions on the surface filled by the liquid; thus, the thermodynamic contact angle is corrected by a multiplicative roughness factor $\left(r_{\mathrm{f}}\right)$ as stated by the following equation:

(27) Adamson, A. W. Physical Chemistry of Surfaces, 6th ed.; John Wiley \& Sons: New York, 1997; Chapter X, Section 5.

(28) Wenzel, R. N. Ind. Eng. Chem. 1936, 28, 988
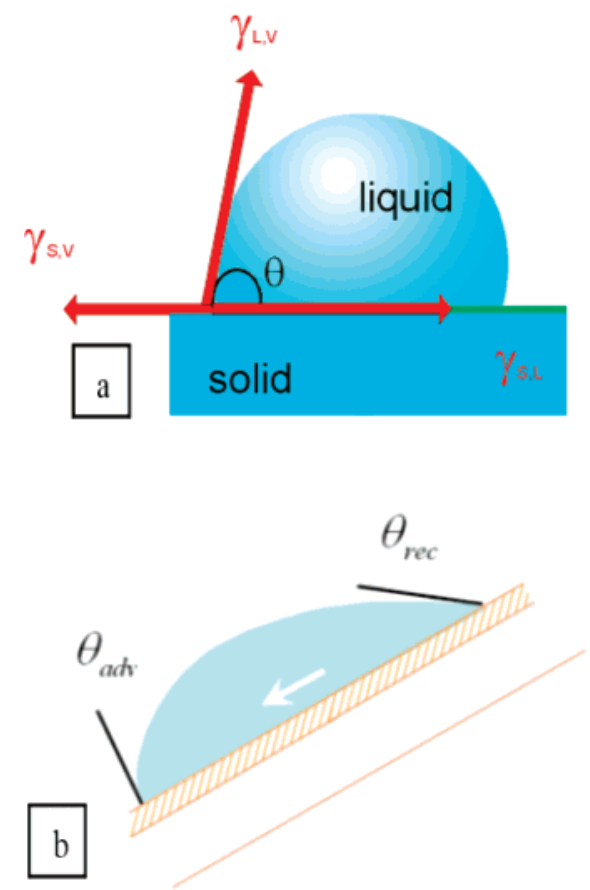

Figure 2. Representation of a drop on a surface describing (a) the contact angle $\theta$ given by the balance between the relative interfacial forces, where $\gamma_{\mathrm{sv}}, \gamma_{\mathrm{sl}}$, and $\gamma_{\mathrm{lv}}$ are the surface tensions at the interface of solid-vapor, solid-liquid, and liquid-vapor, respectively. (b) The advancing and receding contact angle of a water droplet.

$$
\cos \theta_{\mathrm{w}}=r_{\mathrm{f}} \cos \theta
$$

where $\theta$ is the ideal Young contact angle of the liquid on a flat surface and $r_{\mathrm{f}}$ is defined as the ratio of the actual area of the rough surface and the geometric projected area, expressed as a function of the geometric parameters. ${ }^{29}$ Cassie and Baxter's approach $^{30}$ instead considers that liquid sits on the air trapped beneath the drop in the space between the protrusions; hence, the thermodynamic contact angle $\theta_{\mathrm{CB}}$ depends on the fractions of liquid area in contact, respectively, with the solid and with trapped air

$$
\cos \theta_{\mathrm{CB}}=\phi_{s} \cos \theta+\phi_{s}-1
$$

where $\phi_{\mathrm{s}}$ is the area fraction of the liquid-solid contact. Therefore, Cassie-Baxter's or Wenzel state, can be determined by geometric parameters. ${ }^{31}$ The main difference between these two states, as referred by Quéré et al., ${ }^{32}$ relies on the motion of the drop, expected to differ from each state: the Wenzel state is defined as "sticky", as the drop remains pinned to the surface, difficult to move, while the Cassie-Baxter state is described as a "slippery" surface, where the drop beads on such surfaces and there is little resistance to drop attachment or placement. Nevertheless, a high contact angle does not imply superhydrophobic properties, ${ }^{33,34}$ in fact, water contact angle alone is not enough for the evaluation of superhydrophobicity. Further criteria for the measure of hydrophobicity is given by contact angle hysteresis $(\Delta \theta)$ and sliding angles $(\mathrm{SA}, \alpha)$. $\mathrm{CAH}$ is a measure of the heterogeneity of the surface that arises from molecular interactions between liquid

(29) Patankar, N. A. Langmuir 2004, 20, 8209.

(30) Cassie, A. B. D.; Baxter, S, Trans. Faraday Soc. 1944, 40, 546

(31) Oner, D.; McCarthy, T. J. Langmuir 2000, 16, 7777.

(32) Querè, D.; Lafuma, A.; Bico, J. Nanotechnology 003, 14, 1109

(33) Chen, W.; Fadeev, A. Y.; Hsieh, M. C.; Öner, D.; Youngblood, J.; McCarthy, T. J. Langmuir 1999, 15, 3395.

(34) Marmur, A. Langmuir 2004, 20, 3517-3519. 


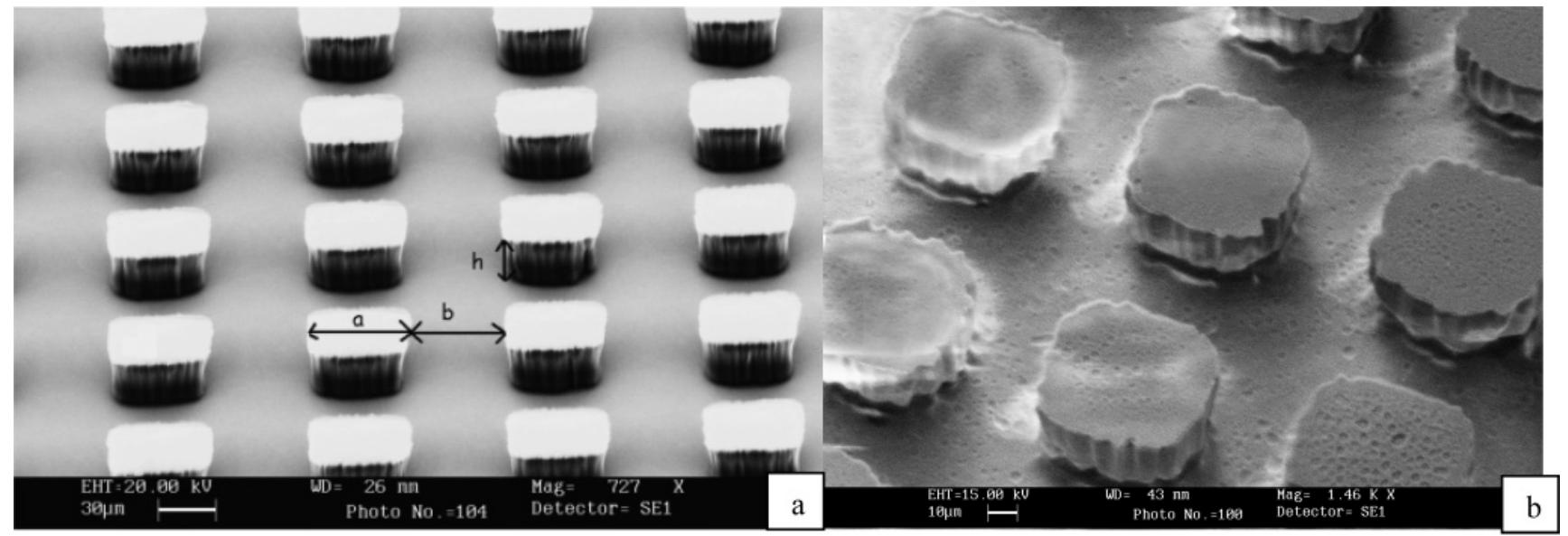

Figure 3. (a) SEM image of a regular array of SU-8 square pillars of size $a \times a$, height $h$ and spacing $b$. (b) SEM image of simple microstructured PDMS pillars, with height of $25 \mu \mathrm{m}$.

and solid and roughness. ${ }^{35}$ Mathematically, it is given by the difference between the advancing contact angle $\left(\theta_{\mathrm{a}}\right)$ and the receding contact angle $\left(\theta_{\mathrm{r}}\right)$ indicated in Figure 2.

SA is a measure of how spontaneously a water droplet will move on the surface ${ }^{36}$ and is commonly employed to assess the dynamic hydrophobicity of a solid surface. ${ }^{32,37}$ Another issue of wetting behavior is the three-phase (solid-liquid-air) contact line. Hysteresis can be caused by a stable contact line, as the droplet placed on the surface comes to rest at a local energy minimum and does not easily roll away. An approach to destabilize the contact line involves random rough hydrophobic surfaces, ${ }^{38}$ so a drop can continuously advance and recede at different contact line points, sliding easily of the surface.

\section{Experimental Section}

Preparation of SU-8 Masters. Photolithography is employed in order to obtain a negative mold for PDMS replication. Patterns were prepared using SU-8 2010, purchased from MicroChem Corp., on silicon wafer substrates. First, the substrates were cleaned by ultrasonication for $10 \mathrm{~min}$ in acetone and $10 \mathrm{~min}$ in 2-propanol and subsequently dried with $\mathrm{N}_{2}$ airflow. The $\mathrm{SU}-8$ process procedure we followed is reported in the manual from MicroChem Corp. ${ }^{39}$ Each sample was spun at an acceleration such as to obtain the desired thickness of ca. $25 \mu \mathrm{m}$. The patterning was carried out by using the Karl Suss MJ B3 mask-aligner with UV illumination and a photomask containing the patterns. Postexposure bake (PEB) was performed on a hot plate for ultimate cross-linking of the resist and allowed to cool down in order to improve adhesion of SU-8 to the substrate. The wafers were then developed by immerging them in SU-8 developer and 2-propanol. Figure 3a shows an SEM image of the microstructured SU-8 pillars.

Preparation of PDMS Samples. Polydimethylsiloxane (PDMS) (Sylgard 184), an elastomeric material composed of an elastomer base and a curing agent, ${ }^{40}$ widely used in soft-lithography, ${ }^{41}$ was purchased from Dow Corning (Midland, MI). Samples were generally prepared from a 10:1 mixture (by weight) of prepolymer and curing agent, poured onto the SU8 masters, and left to settle for $10 \mathrm{~min}$, so that the trapped air bubbles could emerge to the surface. After

(35) Patankar, N. A. Langmuir 2004, 20, 8209-8213.

(36) Miwa, M.; Nakajima, A.; Fujishima, A.; Hashimoto, K.; Watanabe, T. Langmuir 2000, 16, 5754.

(37) McHale, G.; Shirtcliffe, N. J.; Newton, M. I. Analyst 2004, 129, 284

(38) Chen, W.; Fadeev, A. Y.; Hsieh, M. C.; Öner, D.; Youngblood, J. P.; McCarthy, T. J. Langmuir 1999, 15, 3395.

(39) Data sheets, SU-8 photoresist formulations 2-25. http://www.microchem.com/products.

(40) Product data sheet, Sylgard 184 silicone elastomer, http://www.dowcorning.com.

(41) Xia, Y.; Rogers, J. A.; Paul, K. E.; Whitesides, G. M. Chem. Rev. 1999, 99, 1823. the removal of all the air bubbles, the mixture was put into an oven $\left(140{ }^{\circ} \mathrm{C}\right)$ for curing for $15 \mathrm{~min}$. After curing, the PDMS sample was gently peeled from the mold.

The h-PDMS solution was prepared mixing $3.4 \mathrm{~g}$ of trimethylsiloxy-terminated vinylmethylsiloxanedimethylsiloxane (VDT-731; ABCR, Karlsruhe, Germany), $18 \mu \mathrm{L}$ of a Pt catalyst (platinum divinyltetramethyldisiloxane, SIP6831.1; ABCR, Karlsruhe, Germany), and $5 \mu \mathrm{L}$ of a modulator (2,4,6,8-tetramethyltetravinylcyclotetrasiloxane; Sigma Aldrich, St Louis, MO). A 1 g portion of methylhydrosiloxane-dimethylsiloxane (HMS-301; ABCR, Karlsruhe, Germany) was added, after degassing the mixture for 1-2 min, and the mixture was gently stirred subsequently poured onto the master. After baking at $60{ }^{\circ} \mathrm{C}$ for $30 \mathrm{~min}$, Sylgard 184 PDMS was poured onto the h-PDMS layer and cured at $60^{\circ} \mathrm{C}$ for $1 \mathrm{~h}$. Then, the negative template was peeled off from the master

Plasma Treatment. The microstructured PDMS samples were treated in $\mathrm{CF}_{4}$ plasmas generated in a RIE IONVAC inductively coupled (ICP) plasma reactor (PGF 600 RF HUTTER; plasma conditions $150 \mathrm{~W}, 40 \mathrm{mTorr}$ ), operating at a wavelength of the plasma of $1.88 \mu \mathrm{m}$, in order to obtain the submicrometer features on the top of the pillars. Samples were initially exposed to an argon plasma pretreatment (plasma conditions $200 \mathrm{~W}, 40 \mathrm{mTorr}$ ) for 5 min and subsequently to $\mathrm{CF}_{4}$ plasma, with varying exposure times.

Characterization. The samples were characterized using electron microscopy, AFM microscopy, and contact angle measurements. For electron microscopy, the microstructure was observed and evaluated using a LEICA STEREOSCAN 440 SEM operating at 15 $\mathrm{kV}$.

AFM measurements were carried out in air, in tapping mode, using a SMENA MT-DTA atomic force microscope, to analyze roughness of the submicroroughened structure. Software within the AFM was used to calculate average roughness $\left(R_{\mathrm{a}}\right)$ and root-meansquare (rms) roughness on an average of three different images on the sample. The wavelength of the periodicity was determined by performing a numerical fast Fourier transform (FFT) analysis on the AFM images.

Contact angles of water on the different substrates were measured in laboratory atmosphere at room temperature using the sessile drop method of a contact angle goniometer (Dataphysics OCA 20). A drop of $3 \mu \mathrm{L}$ was deposited on the surface, and each reported angle is calculated as the average of six measurements in different points on the sample. The advancing and receding contact angle were read by slowly increasing and decreasing the size of the drop by removing $3 \mu \mathrm{L}$ from a drop of ca. $5 \mu \mathrm{L}$.

Sliding angle measurements were carried out using a tiltable plate to which the sample was fixed. A drop of $10 \mu \mathrm{L}$ was applied to the sample and the plate was slowly inclined until the drop started to move. The measurement was calculated as the average of six individual measurements. 
Table 1. Geometric Parameters of the Microstructured PDMS Pillars with Width $a$ and Spacing $b$

\begin{tabular}{ccc}
\hline sample & width, spacing $(\mu \mathrm{m})^{a}$ & fill factor $^{b}$ \\
\hline 1 & $a=28, b=28$ & 0.25 \\
2 & $a=42, b=42$ & 0.25 \\
3 & $a=49, b=49$ & 0,25 \\
4 & $a=49, b=28$ & 0.4 \\
5 & $a=42, b=14$ & 0.56 \\
6 & $a=70, b=10$ & 0.76 \\
7 & flat & 1
\end{tabular}

${ }^{a}$ The height $(h)$ was $25 \mu \mathrm{m} .{ }^{b}$ The fill factor is defined as the percentage of the patterned area with respect to the total area, calculated as $a^{2} /(a$ $+b),{ }^{2}$ where the fill factor of a flat sample was obviously 1 .

\section{Results and Discussion}

Topography is a key factor when related to hydrophobicity, enhancing the effect of surface chemistry within superhydrophobicity. Clearly, combining appropriate surface roughness with low-surface-energy materials is important for the fabrication of superhydrophobic surfaces. Hence, a material with low surface energy, like PDMS, was chosen, due to its biocompatibility, intrinsic hydrophobic behavior, and low water absorption. Moreover, PDMS facilitates replication of complex topographic patterns and exhibits a contact angle on a flat surface of $115^{\circ}$. In order to attain a monoroughness, microstructured PDMS pillars were obtained by replication of a negative mold of an SU-8 master (see Experimental Section). Various templates of roughness were produced, changing the value of the fill factor, defined as the ratio of the patterned area to the total area, in order to evaluate the latter's influence within CA measurements. A total of six different pillars of different geometry parameters surfaces, the dimensions of which are listed in Table 1, were chosen.

Three kinds of pillars with the same fill factor but different spacing were chosen in order to see differences owing to spacing and dimensions, while the other pillars were chosen to vary the fill factor value. The images of the regular arrays of SU-8 square pillars and microstructured PDMS surfaces were viewed under scanning electron microscopy (SEM) using a LEICA STEREOSCAN 440 SEM operating at $15-20 \mathrm{kV}$, as shown in Figure 3a,b.

By varying the geometry of width $(a)$ and spacing $(b)$, but keeping the fill factor value constant, values of the contact angles increased as the dimensions of the pillars and spacing decreased, as shown in Figure 4a. Thus, as the dimensions of the pillars and spacing became smaller, and a more fakir-like configuration was assumed by the structure, water droplets were pinned on the top of the pillars without seeping between them, as confirmed by hysteresis values. As the fill factor was increased, by varying the geometric parameters, lower contact angles were achieved, as shown in Figure 4b, presumably due to a decrease of the formation of air pockets underneath the water droplet. This assumption is reinforced by a decrease in the hysteretic wetting behavior of the monostructured surfaces with a decrease of the fill factor value, as shown in Figure 4b, though these values were not alone enough to provide a slippery state. The water drops, in fact, were pinned to the monostructured surface. As shown in Figure $4 b$, there is also an abrupt change of the hysteresis to lower values with the decrease of the fill factor, due to a major spacing between the pillars and presumably to the formation of air pockets preventing the seeping of the water.

These results suggested that simple microstructured surfaces are unable to obtain a superhydrophobic behavior in untreated PDMS slabs, and an additional roughness as well as a low surface
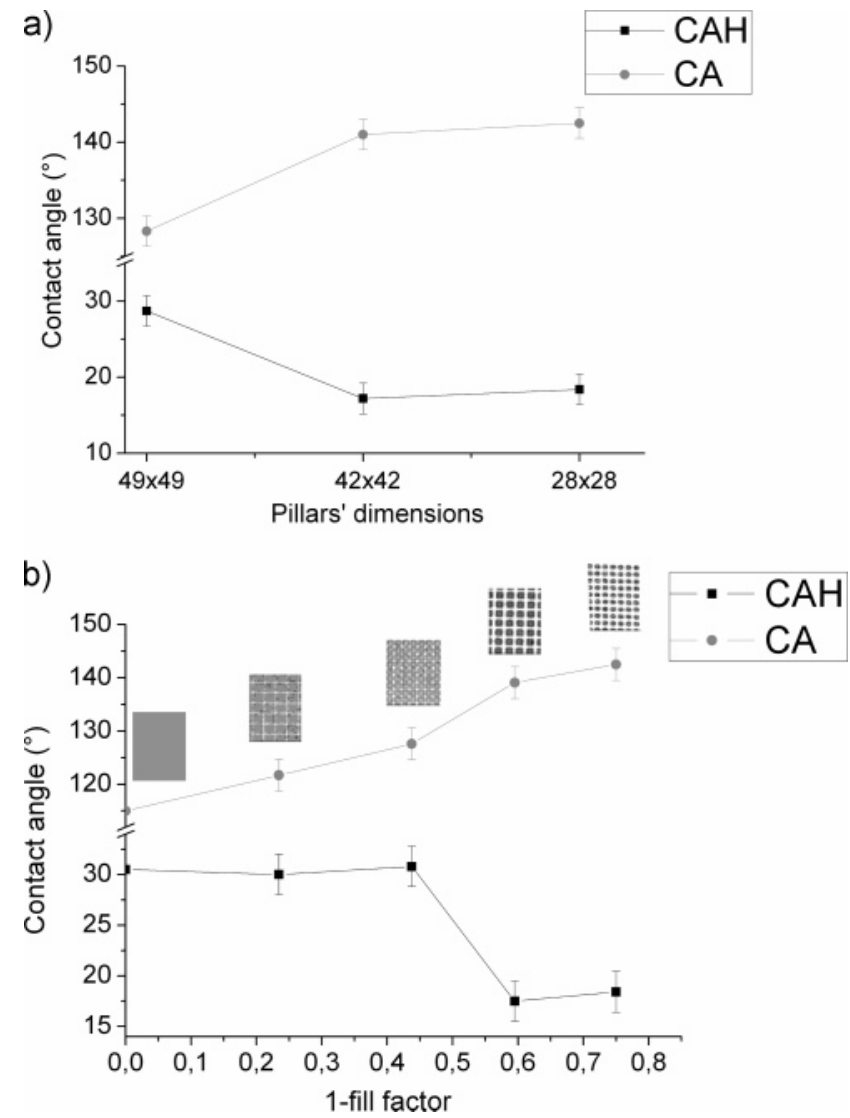

Figure 4. (a) Plot of the contact angle CA and hysteresis CAH as a function of the same fill factor but with pillars of different dimensions. With smaller pillars and spacing, the surface assumed a more fakir-like structure, so values of contact angles increased with a decrease of hysteresis; therefore, pillars of dimensions $28 \times$ 28 were chosen to be compared with the other structures. (b) Plot of the contact angle $\mathrm{CA}$ and hysteresis $\mathrm{CAH}$ as a function of 1-fill factor (air fraction), showing an increase of contact values and a decrease of hysteresis by increasing the air fraction. Contact angles value and hysteresis of a flat surface, start from $115^{\circ}$ and $30^{\circ}$, respectively. An abrupt change of data 3 and 4 is due to the change of spacing, which presumably gave rise to the presence of air pockets and, therefore, to the prevention of water intruding between the pillars, thus lowering hysteresis.

energy component have to be superimposed..$^{42,29}$ Consequently, we increased the roughening on the top of the pillars through micro- and nanostructuring the surface, by employing a $\mathrm{CF}_{4}$ glow discharge plasma. The plasma treatment process can be summarized in two main steps. First, an Ar-plasma pretreatment realized the formation of stable free radicals on the polymer surface. Second, a $\mathrm{CF}_{4}$ plasma enabled the formation of microand submicrometer structures, allowing the fluorine plasma component to react with the free radicals and to deposit a low surface energy thin fluorination film. ${ }^{43,44}$ By varying plasma conditions, different degrees of the second scale of roughness were obtained on the monostructured pillars, easily controlling the diameter and area density of the submicrometer features. The second scale of roughness and periodicity increased upon increasing plasma exposure time. Indeed, we observed the formation of submicrometer structures, the roughness of which ranged from a few hundred nanometers up to $3.5 \mu \mathrm{m}$ and the quasiperiodicity of which ranged from $424 \mathrm{~nm}$ up to $1.88 \mu \mathrm{m}$,

(42) Herminghaus, S. Europhys. Lett. 2000, 52 (2), 165-170.

(43) Szmigiel, D.; Domański, K.; Prokaryn, P.; Grabiec, P.; Sobczak, J. W. Appl. Surf. Sci. 2006, 253, 1506.

(44) Yan, Y. H.; Chan-Park, M. B.; Yue, C. Y. Langmuir 2005, 21, 8905. 


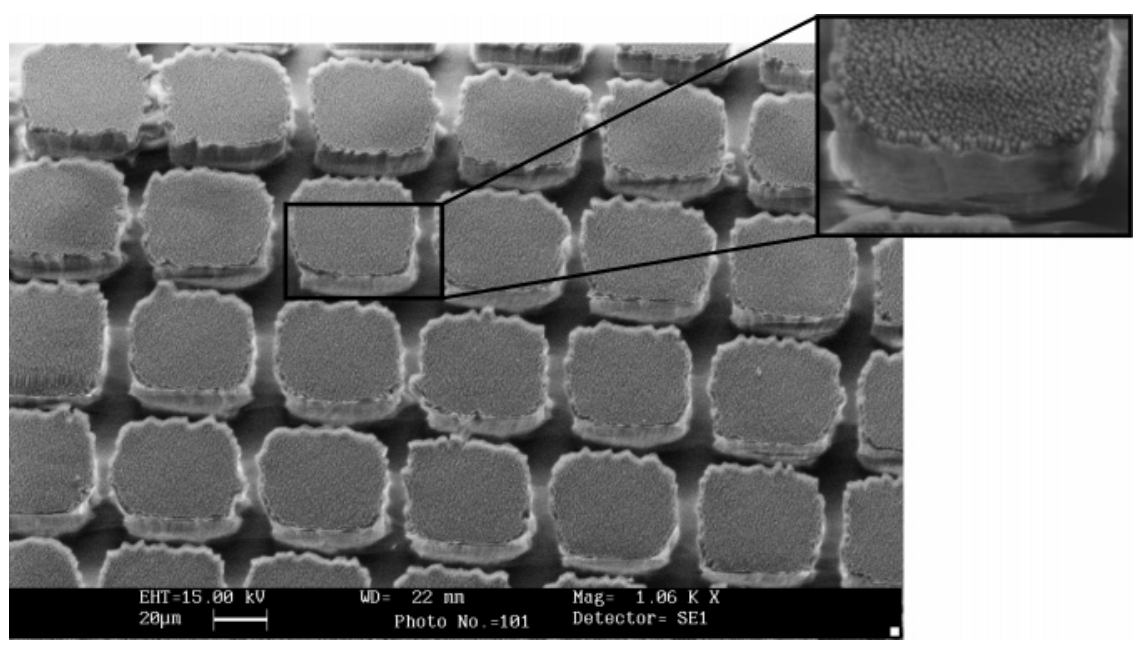

Figure 5. SEM images of double-structured PDMS pillars with width of $42 \mu \mathrm{m}$ and spacing of $14 \mu \mathrm{m}$; a further magnification showing the random distribution of the submicron features is reported in the inset.
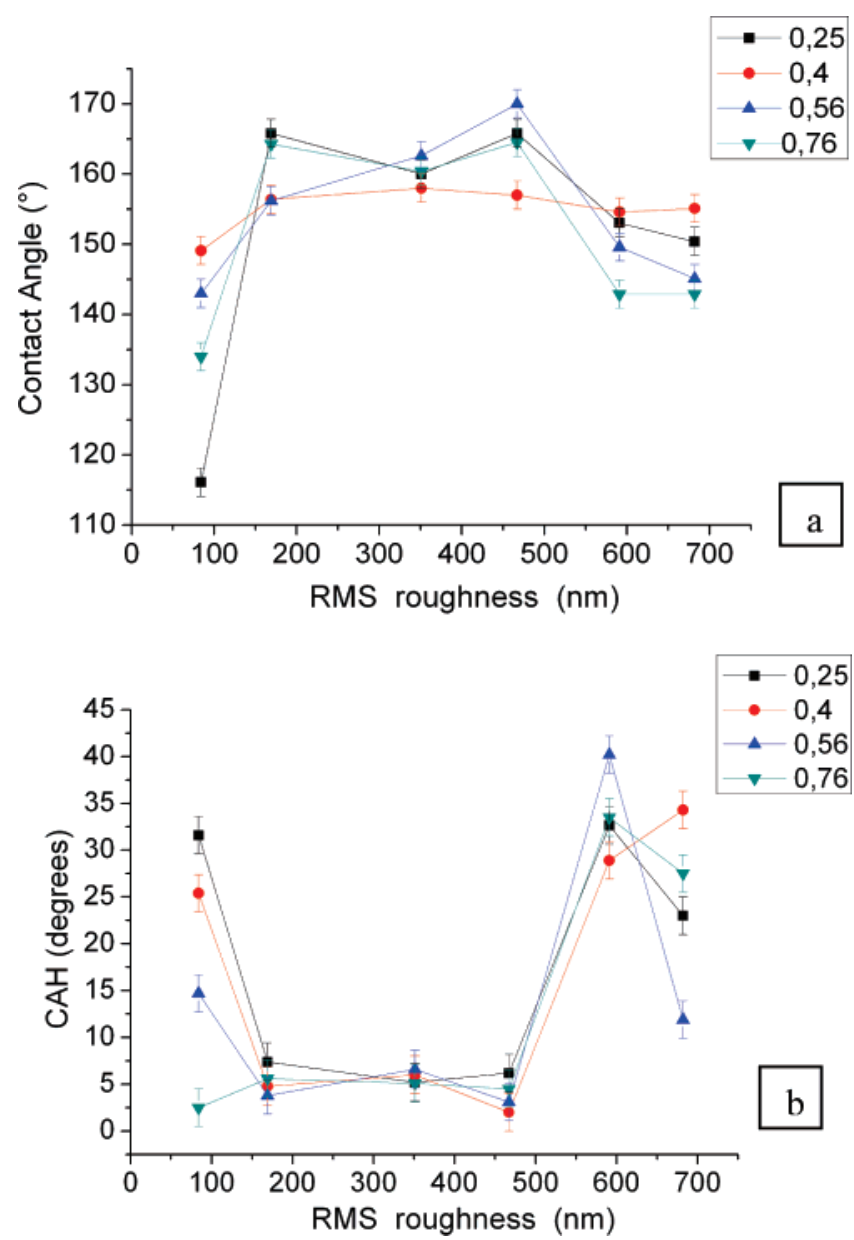

Figure 6. Plot of the variation of the contact angle (a) and hysteresis (b) of the six treated samples related to the surface roughness as a function of different plasma exposure times. The insets show photographs of water droplet on the different surfaces.

in proportion with treatment time. The SEM image, reported in Figure 5, shows the typical micro- and nanoroughness superimposed on the top surface of each pillar obtained through this process.

As clearly seen from the data reported in Figure 6, with the addition of the second submicro- and nanoscale roughness on the prepatterned microscale array, the contact angle increases systematically as the surface roughness increases, with an improvement of superhydrophobic properties. Aside from the higher contact angle values, we observed the dynamic behavior of water on the plasma-treated double-scale roughened pillars, measuring the advancing and receding angles and hysteresis to assess wettability. Figure $6 \mathrm{~b}$ shows the variation of the water contact angle and contact angle hysteresis as a function of roughness of the double-structured pillars. The plot indicates that the $\mathrm{CAH}$ angles were significantly lower for the doublestructured pillars than for the untreated ones. However, these values were sensitive to differences in the second scale roughness, becoming smaller as the roughness approached values that ranged from 100 to $500 \mathrm{~nm}$. This decrease in hysteresis may be a result of an increase of pillars density, which brings a decrease of the fraction of solid in contact with the liquid, switching from Wenzel's state and leading to a slippery one.

For plasma-treated surfaces that exhibited a roughness lower than $100 \mathrm{~nm}$ and higher than $500 \mathrm{~nm}$, water droplets were strongly pinned to the surface, corresponding typically to the Wenzel regime. This behavior was emphasized by the high hysteresis, shown in Figure 6b. A further increasing of the roughness conferred a lowering of hysteresis, presumably due to a deeper etching and, therefore, again a change of the topology. In effect, for high roughness values, this is presumably due to the seeping of the water droplets between the larger spaced submicro- and nanostructures, while for lower roughness the nanostructures where not high enough to prevent intrusion of water. These morphologies caused the drop boundary to obtain sufficient contact to pin the drop. Instead, on the remaining plasma-treated samples, especially within a roughness of 150-500 nm, we observed a rapid switching from Wenzel's state to Cassie's, as evidenced by the decrease in the hysteresis down to less than $4^{\circ}$, within which water droplets remained nearly spherical and did not easily wet the surface, as shown in Figure 7a. Besides, hysteresis within the range roughness of $150-500 \mathrm{~nm}$ was less influenced by the geometrical parameters of the pillars. It seems that, in this range, when the fill factor is combined with a second scale of roughness, contact angle's and hysteresis values are not influenced by different dimensions and distances between pillars, exhibiting a slippery effect. Therefore, it is clearly seen that the hierarchical roughness dramatically increases the CA and efficiently decreases the CA hysteresis. In terms of the contact line formed at the interfaces between solid, liquid, and air, it seems that the hierarchical structure is capable of breaking the continuity of the three-phase contact line at the solid-liquid interface, resulting in a lower value of hysteresis. The roughness 

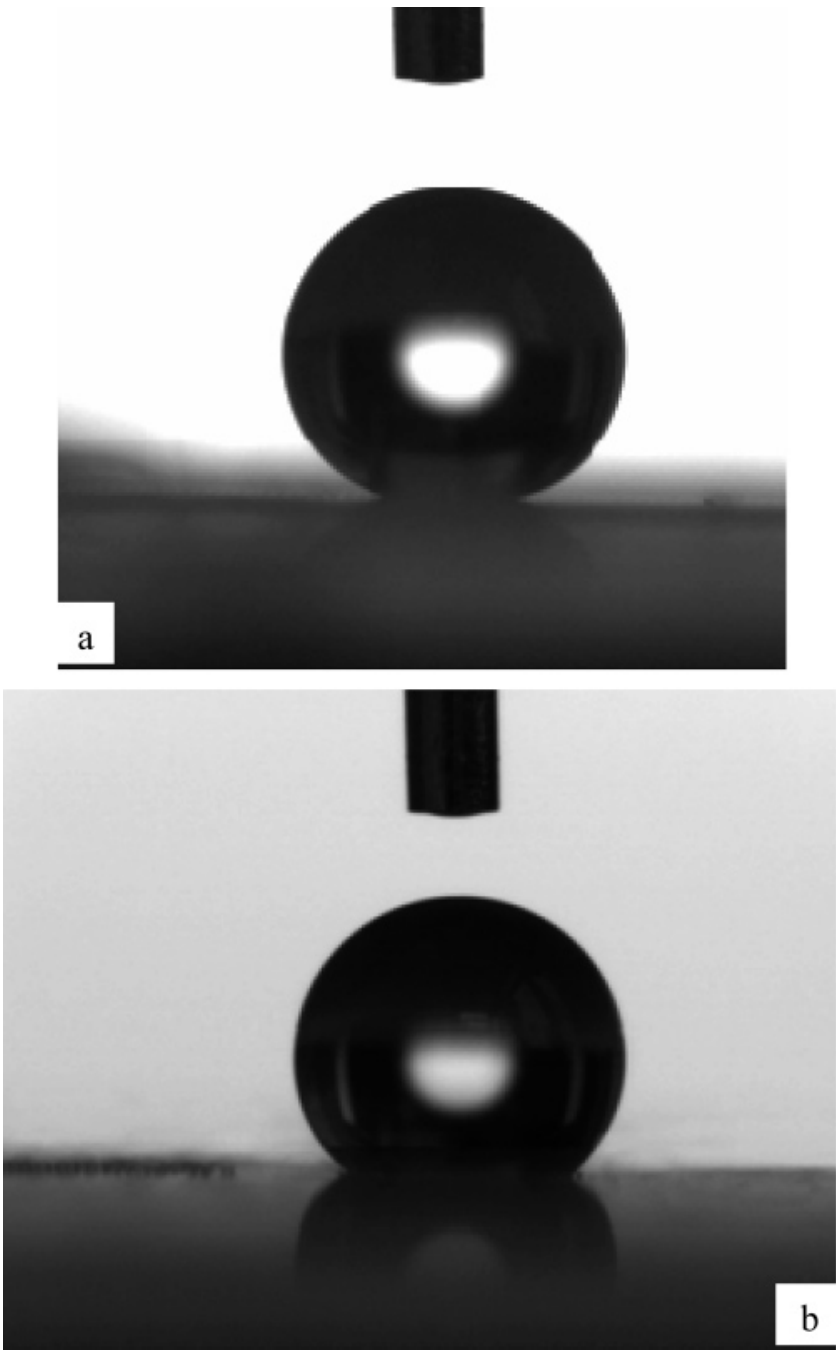

Figure 7. (a) Contact angle measurement on the rough etched prepatterned PDMS surfaces. (b) Contact angle measurement of the drop pinned to the etched surface but not on the microscale pattern, showing the enhancement of hydrophobicity.

range between 100 and $500 \mathrm{~nm}$ provided an effective means of trapping sufficient air to form air pockets and obtain a slippery effect. For surfaces with higher roughness, the contact angle hysteresis increases as the surface roughness increases; therefore, the contact interface area increased due to the increase in surface roughness, with eventual water intrusion.
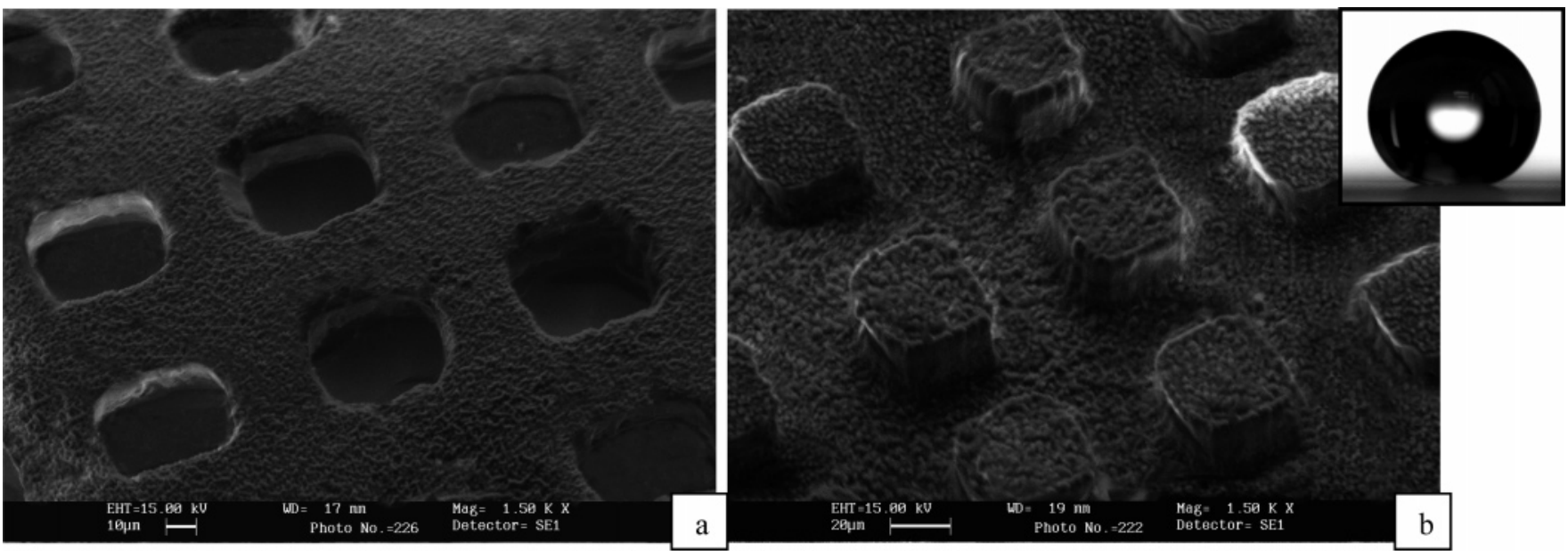

Figure 8. SEM images of (a) the negative and (b) the positive replica of the double-structured PDMS pillars with roughness of 450 nm In the inset on the right, the contact angle measurement of the drop on the positive replica, the value of which was about $15^{\circ}$ lower with respect to the original structure.
A further check of different active wetting regimes was given by measuring the sliding angles with a water drop of $10 \mu \mathrm{L}$ on a tilting table. The surface was gradually inclined from its horizontal alignment until the water droplets started to slide or to roll off. It was observed that the water droplets assumed a more spherical shape on surfaces with roughness ranging between 100 and $500 \mathrm{~nm}$, rolling off more spontaneously, even with a slight movement of the sample position. Nonetheless, a considerable difference between the dual structured surface and a surface without micropatterning was observed, as shown in Figure $7 a, b$.

The dual structure and the simple roughness due to plasma etching without micropillars showed unlike wetting properties. These differences were emphasized by observing the rolling behavior of water droplets once placed on them. Water droplets placed upon the double-structured sample simply rolled across the patterned surface, with a very small tilt, but once past the rim of the pattern, they came to a rest, being pinned to the nano-, submicrometer-roughened surface. Obviously this was due to the lack of the presence of the micropatterned structure, as shown in Figure 7b, which, owing to the spacing between the pillars, introduced air pockets, and thus a Cassie state, despite the submicrometer roughening still present on the surface. This strongly confirmed the enhancement of hydrophobicity due to a combination of the controlled regular double microhierarchical structure compared to a single random microroughness. From the above analysis, it can be concluded that a dual structured contact is needed for droplet motion.

In order to analyze the effect due to the superposition of the hierarchical roughness of the structures separated from the chemical effect given by the fluorination film, we doublereplicated the sample, obtaining a positive replica. A mold that represents the negative of the original pattern, shown in Figure 8a, was obtained by casting h-PDMS over the original master structure. The resulting solidified negative master was easily stripped from the original structure. For the subsequent replication of the negative mold, an antistick monolayer of octadecyltrichlorosilane (OTS) was evaporated on the elastomeric replica and then used to reproduce the original structure, by casting liquid PDMS. After curing at $60^{\circ} \mathrm{C}$ for $1 \mathrm{~h}$, the positive PDMS replica was successfully peeled off from the master, as shown in Figure 8b, resulting in a corresponding topographic surface structure of the original structure.

A double replica actually confirmed an increase of the contact angle due to the double roughness with respect to the monor- 
oughness, without chemical effects. Although lower values of contact angles ranging from $15^{\circ}$ were observed, obviously due to the lack of the chemical contribution, still the contact angles achieved were higher than those without a double structure. This confirmed that these superhydrophobic polymeric surfaces mimicked the well-known dual-scale roughness of lotus leaves, along with the chemical contribution, without the need of an additional coating. Another important result was that the superhydrophobic double-structured surfaces we fabricated were extremely stable under ambient conditions. The treated samples showed repeatability and durability, demonstrating superhydrophobic characteristics within months from the treatment, still showing the slippery effect on the water droplets, undoubtedly due to the intrinsic hydrophobic behavior of PDMS.

\section{Conclusion}

In conclusion, we have fabricated a superhydrophobic engineered surface mimicking a structure similar to that of lotus leaves, by employing a simple etching method. The formed superhydrophobicity results from a hierarchical dual-scale roughened structure composed of micro- and nanopillars.
Moreover, we have investigated how superimposing submicroand nanostructures on a monoroughness is an important factor in reducing the wettability of hydrophobic surfaces, observing water droplets rolling across the surface and coming to rest when beyond the micropatterned surface. This enhanced the importance of a double-roughness structure, as it showed a more slippery behavior only within the prepatterned structures with a dualstructured surface, whereas the drops were pinned to the border of the pattern with a monoroughness due to the change of surface geometry. Furthermore, an important aspect of the plasma process was the fluorination of the surface, which made unnecessary a further coating. Therefore, a hierarchical rough structure could be an effective structure for moving droplet application, as hysteresis was strongly reduced, allowing control of droplet motion by introducing low sliding angles.

Acknowledgment. This research has been partially supported by MIUR, project FIRB Synergy, and iGuzzini. The authors would also like to thank Eliana D'Amone and Claudia Piliego for useful technical support.

LA702764X 